\title{
Retraction Note to: Effects of microRNA-21 on Nerve Cell Regeneration and Neural Function Recovery in Diabetes Mellitus Combined with Cerebral Infarction Rats by Targeting PDCD4
}

\author{
Yun-Bao Guo ${ }^{1} \cdot$ Tie-Feng $\mathrm{Ji}^{2} \cdot$ Hong-Wei Zhou ${ }^{2} \cdot$ Jin-Lu Yu ${ }^{1}$ \\ (C) Springer Science+Business Media, LLC, part of Springer Nature 2021, corrected publication 2021
}

Retraction Note to: Mol Neurobiol (2018) 55:2494-2505.

https://doi.org/10.1007/s12035-017-0484-8

The authors have retracted this article. After publication they became aware that an incorrect method of statistical analysis had been used (one-way ANOVA instead of repeated measures ANOVA), rendering the conclusions unreliable. The Editor has invited the authors to re-analyse their data and submit a new manuscript for consideration for publication.

All authors agree with this retraction.

Publisher's Note Springer Nature remains neutral with regard to jurisdictional claims in published maps and institutional affiliations.

The online version of the original article can be found at https://doi.org/ 10.1007/s12035-017-0484-8

Jin-Lu Yu

jinluyu@hotmail.com

1 Department of Neurosurgery, The First Hospital of Jilin University, No. 1, Xinmin Road, Chaoyang District, Changchun 130021, Jilin Province, People's Republic of China

2 Department of Radiology, The First Hospital of Jilin University, No. 1, Xinmin Road, Chaoyang District, Changchun 130021, People's

Republic of China 